\title{
Ferromagnetic properties of charged vector bosons condensate in the early universe
}

\author{
Gabriella Piccinelli \\ Centro Tecnológico, FES Aragón, Unversidad Nacional Autónoma de México \\ Av. Rancho Seco S/N, Bosques de Aragón, Nezahualcóyotl \\ Estado de México 57130, Mexico \\ email: itzamna@unam.mx
}

\begin{abstract}
Bose-Einstein condensation in the early universe is considered. The magnetic properties of a condensate of charged vector bosons are studied, showing that a ferromagnetic state is formed. As a consequence, the primeval plasma may be spontaneously magnetized inside macroscopically large domains and primordial magnetic fields can be generated.
\end{abstract}

Keywords. Early universe, magnetic fields, elementary particles.

\section{Introduction}

Cosmological Magnetic fields. Magnetic fields seem to be pervading the entire universe: they have been observed in galaxies, clusters and high redshift objects (for observational reviews see, e.g., Kronberg 1994 and Carilli \& Taylor 2002). Although at present there is no conclusive evidence about their origin, their existence in the early universe cannot certainly be ruled out. They use to be appreciated in cosmology since they help to solve some problems, in particular in the baryogenesis process (e.g., Sánchez, Ayala \& Piccinelli 2007 and references therein and Piccinelli \& Ayala 2004, for a review).

The determination of the physical processes able to generate magnetic fields represents a long standing cosmological problem. The general approach is to identify mechanisms for the generation of seed fields which can later be amplified into fields on larger scales, but nowadays no completely successful mechanism has been proposed.

Bose-Einstein condensates (BEC). Another interesting phenomenon that may be present in the early Universe is Bose-Einstein condensation: a quantum phenomenon of accumulation of identical bosons in the same state, which is their lowest energy (zero momentum) state. Under these conditions they behave as a single macroscopic entity described by a coherent wave function rather than a collection of separate independent particles. Even though Bose-Einstein condensation had been foreseen long time ago (1925), it took seventy years to make the first experimental observation, which was performed in a dilute gas of rubidium (Anderson et al. 1995). Difficulties in performing this observation were created by the extremal conditions necessary for the condensation. Indeed, the BoseEinstein condensation takes place when the inter-particle separation is smaller than their de Broglie wavelength, $\lambda_{d B} \sim 2 \pi / \sqrt{2 m T}$, so the system must be cooled down to a very low temperature at ordinary densities. In the early universe, the existence of condensates depends on an interplay between density and temperature (see Dolgov, Lepidi \& Piccinelli 2010, for a discussion on the conditions for which condensation could take place).

In the recent years the study of BEC became an active area of research in different fields of physics from plasma and statistical physics (for a review see Pethick \& Smith 
2002 and references therein) to astrophysics and cosmology. For instance, neutral scalar BEC have been proposed as dark matter (Ji \& Sin 1994, Matos \& Guzmán 1999).

The presence of charged BEC has interesting consequences in gauge field theories. For instance, in Dolgov, Lepidi \& Piccinelli (2009), electrodynamics of charged fermions and condensed scalar bosons was considered. The screening of impurities in such plasma was found to be essentially different from the case when the condensate is absent. A similar problem was considered also in the framework of an effective field theory (Gabadadze \& Rosen (2008), (2009), (2010)), analyzing the thermodynamical properties of the system and focusing on the astrophysics of helium white dwarfs. The possible condensation of helium nuclei, previous to crystallization, would affect the cooling process and leave observational signatures.

The condensation of gauge bosons of weak interactions was considered in the pioneering papers by Linde (1976), (1979), where it was argued that, at sufficiently high leptonic chemical potential, a classical $W_{j}$ boson field could be created and, under certain conditions, there could be no symmetry restoration in the early universe.

As we have seen in the previous applications, the condensate can be made of either scalar or vector bosons. In both cases, bosons are in the lowest energy state, but in the vector case they have an additional degree of freedom: spin. The state of rest and identicalness inherent to the condensed particles suggests that they could present an ordered configuration of their spin magnetic moments.

In this work we consider the BEC of charged $W$-bosons, which may be formed if the cosmological lepton asymmetry happened to be sufficiently high, i.e. if the chemical potential of neutrinos was larger than the $W$ boson mass at this temperature, and study its magnetic properties. We concentrate on the condensation below electroweak phase transition, although the phenomenon could also be considered above it.

\section{The model}

We consider a simple example of electrically neutral plasma made of fermions (electrons and neutrinos), gauge and scalar bosons, with zero baryonic number density but with a high leptonic one. This implies a (large) chemical potential for leptons and $\mathrm{W}$ bosons and, consequently, the formation of a charged vector BEC. For simplicity we work with only one family of leptons, but this does not influence the essential features of the result. Quarks may be essential for the condition of vanishing of all gauge charge densities in plasma and for the related cancellation of the axial anomaly but we work in the lowest order of the perturbation theory, where the anomaly is absent.

A caveat for a large lepton asymmetry arises from the big bang nucleosynthesis (BBN) with strongly mixed neutrinos. It is shown in Dolgov et al. (2002) that leptonic chemical potentials of all neutrino flavors are restricted by $\left|\mu_{\nu} / T\right|<0.07$ at the BBN epoch. However, it should be noted that the entropy release from the electroweak epoch down to the BBN epoch diminishes the lepton asymmetry, by the ratio of the particle species present in the cosmological plasma at these two epochs, which is approximately 10 . This helps to alleviate the bound and to allow favorable conditions for condensation in the early universe, still compatible with BBN (see Dolgov, Lepidi \& Piccinelli 2010, for a detailed discussion on the different possibilities).

The essential reactions are the direct and inverse decays of $W: W^{+} \leftrightarrow e^{+}+\nu$. The equilibrium with respect to these processes imposes the equality between the chemical potentials: $\mu_{W}=\mu_{\nu}-\mu_{e}$, and the condition of electroneutrality reads: $n_{W^{+}}-n_{W^{-}}-$ $n_{e^{-}}+n_{e^{+}}=0$. 
Considering the Lagrangian of the minimal electroweak model, we derive the equations of motion that allow to take into account the spin-spin interactions of the vector bosons.

\section{Spin-spin interactions of the condensed bosons}

The spins of the individual vector bosons can be either aligned or anti-aligned, forming, respectively, ferromagnetic and anti-ferromagnetic states, see, e.g., Pethick \& Smith (2002). The realization of one or the other state is determined by the spin-spin interaction between the bosons. In the lowest angular momentum state, $l=0$, a pair of bosons may have either spin 0 or 2. Depending on the sign of the spin-spin coupling, one of those states would be energetically more favorable and would be realized at the condensation.

In solid state physics, the dominant contribution to the spin-spin interactions comes from quantum exchange effects; for bosons these forces are not essential and the spinspin interaction is determined by the electromagnetic interaction between their magnetic moments and by their self-interactions (Dolgov, Lepidi \& Piccinelli 2010).

Electromagnetic interactions can be found from the analogue of the Breit equation for electrons, which leads to the spin-spin potential:

$$
U_{e m}^{\text {spin }}(r)=\frac{e^{2}}{4 \pi m_{W}^{2}}\left[\frac{\left(\mathbf{S}_{1} \cdot \mathbf{S}_{2}\right)}{r^{3}}-3 \frac{\left(\mathbf{S}_{1} \cdot \mathbf{r}\right)\left(\mathbf{S}_{2} \cdot \mathbf{r}\right)}{r^{5}}-\frac{8 \pi}{3}\left(\mathbf{S}_{1} \cdot \mathbf{S}_{2}\right) \delta^{(3)}(\mathbf{r})\right],
$$

where $\mathbf{S}=-i \mathbf{W}^{\dagger} \times \mathbf{W}$ is the spin operator of vector particles and $m_{W}$ its mass.

To calculate the contribution of this potential into the energy of two $W$-bosons we have to average it over their wave function. In particular, in the condensate case, it is a $\mathrm{S}$-wave function that is angle independent. Hence the contributions of the first two terms in the previous equation mutually cancel out and only the third one remains, which has negative coefficient. Thus the energy shift induced by the spin-spin interaction is:

$$
\delta E=\int \frac{d^{3} r}{V} U_{e m}^{s p i n}(r)=-\frac{2 e^{2}}{3 V m_{W}^{2}}\left(\mathbf{S}_{1} \cdot \mathbf{S}_{2}\right),
$$

where $\mathrm{V}$ is the normalization volume.

Since $S_{\text {tot }}^{2}=\left(S_{1}+S_{2}\right)^{2}=4+2 S_{1} S_{2}$, the average value of $S_{1} S_{2}$ is $S_{1} S_{2}=S_{\text {tot }}^{2} / 2-2$. For $S_{\text {tot }}=2$ this term is $S_{1} S_{2}=1>0$, while for $S_{\text {tot }}=0$ it is $S_{1} S_{2}=-2<0$. Thus, for this interaction, the state with maximum total spin is more favorable energetically and $W$-bosons should condense with aligned spins.

Quartic self-coupling of $W$. Due to its non-Abelian character, the weak sector presents a quartic self-coupling in the Lagrangian:

$$
L_{4 W}=-\frac{e^{2}}{2 \sin ^{2} \theta_{W}}\left[\left(W_{\mu}^{\dagger} W^{\mu}\right)^{2}-W_{\mu}^{\dagger} W^{\mu \dagger} W_{\nu} W^{\nu}\right]=\frac{e^{2}}{2 \sin ^{2} \theta_{W}}\left(\mathbf{W}^{\dagger} \times \mathbf{W}\right)^{2},
$$

where $\theta_{W}$ is the Weinberg angle. It is assumed here that $\partial_{\mu} W^{\mu}=0$ and thus only the spatial 3 -vector $\mathbf{W}$ is non-vanishing, while $W_{t}=0$.

The interaction potential is given by the Fourier transform of this term which, with proper (nonrelativistic) normalization, leads to:

$$
U_{4 W}^{(s p i n)}=\frac{e^{2}}{8 m_{W}^{2} \sin ^{2} \theta_{W}}\left(\mathbf{S}_{1} \cdot \mathbf{S}_{2}\right) \delta^{(3)}(\mathbf{r}) .
$$

Thus, the contribution of the quartic self-coupling of $W$ to the spin-spin interaction has opposite sign to the electromagnetic term and tends to favour an antiferromagnetic state. Nonetheless, in the standard model, the electromagnetic term is dominant. 


\section{Discussion and Conclusions}

Generation of primordial magnetic fields. We have studied the Bose-Einstein condensation of charged weak bosons, driving special attention to the magnetic properties of the condensate. The spin-spin interaction has two contributions: the electromagnetic one - leading to a ferromagnetic state - and a contact quartic self-interaction that produces an antiferromagnetic state. We have found that the former dominates, generating a macroscopically large ferromagnetic configuration. We then expect that the primeval plasma, where such bosons condensed, can be spontaneously magnetized. The typical size of the magnetic domains is determined by the cosmological horizon at the moment of the condensate evaporation. The latter takes place when the neutrino chemical potential, which scales as temperature in the course of cosmological cooling down, becomes smaller than the $W$ mass at this temperature. Large scale magnetic fields created by the ferromagnetism of $W$-bosons might survive after the decay of the condensate due to the conservation of the magnetic flux in plasma with high electric conductivity. Such magnetic fields at macroscopically large scales could be the seeds of the observed larger scale galactic or intergalactic magnetic fields.

Some considerations: The long range interactions between magnetic moments can, in principle, be screened by plasma physics, while the local quartic interaction cannot. This could change the relative strength of these two effects. However, in the broken phase, the problem is reduced to that of pure QED, where it is known that magnetic forces are not screened. On the other hand, the situation is not clear in non-Abelian theory and it could happen that, in the symmetric phase, the screening might inhibit magnetic spinspin interaction. Interactions with relativistic electrons and positrons are neglected. In principle, they could distort the spin-spin interactions of $W$ by their spin or orbital motion and thus destroy the attraction of parallel spins of $W$. However, it looks hardly possible because electrons are predominantly ultra-relativistic and they cannot be attached to any single $W$ boson to counterweight its spin. Finally, the scattering of electrons (and quarks) on $W$-bosons may lead to the spin flip of the latter, but in thermal equilibrium this process does not change the average value of the spin of the condensate.

\section{References}

Kronberg, P. P. 1995, Rep. Prog. Phys., 57, 325; Carilli, C. L. \& Taylor, G. B., 2002, Ann. Rev. Astron. Astrophysics, 40, 319

Sánchez, A. Ayala, A., \& Piccinelli G. , 2007, Phys. Rev., D75, 043004; Piccinelli, G. \& Ayala, A., 2004, in: N. Breton, J. L. Cervantes-Cota, M. Salgado, (eds.), The Early Universe and Observational Cosmology, (Lect.Notes Phys. 646: Springer Verlag), p. 293

Anderson, M., Ensher, J., Matthews, M., Wieman, C., \& Cornell, E., 1995, Science, 269, 198

Dolgov, A. D., Lepidi, A., \& Piccinelli, G, 2010, JCAP, 08, 031

Pethick, C. J. \& Smith, H., 2002, Bose-Einstein condensation in dilute gases, (Cambridge University Press, Cambreidege U.K.)

Ji, S. U. \& Sin, S-J., 1994, Phys. Rev. D50, 3655; Matos, T. \& Guzmán, F. S., 1999, F. Astron. Nachr., 320, 97

Dolgov, A. D., Lepidi, A., \& Piccinelli, G, 2009, JCAP, 02, , 016; Phys. Rev., D80, 125009

Gabadadze, G. \& Rosen, R. A., 2008, JCAP, 10, 030; 2009, JCAP, 02, 016; 2010, JCAP, 04, 028

Linde, A. D., 1976, Phys. Rev., D14, 3345; 1979, Physics Letters, 86B, 39

Dolgov, A. D., Hansen, S. H., Pastor,S., Petcov, S. T., Raffelt, G. G., \& Semikoz, D. V., 2002, Nucl. Phys., B632, 363 